\title{
Osteocytes and their messengers as targets for the treatment of multiple myeloma
}

Jesus Delgado-Calle $e^{1,2}$

${ }^{1}$ Department of Anatomy and Cell Biology, Indiana University School of Medicine, ${ }^{2}$ Roudebush Veterans Administration Medical Center, Indianapolis, Indiana, USA.

\section{Corresponding author's information:}

Jesus Delgado-Calle, Ph.D.

Department of Anatomy and Cell Biology, Indiana University School of Medicine

635 Barnhill Drive, MS5045L; Indianapolis, IN 46202, USA.

Phone: 317-274-2323

Fax: 317-278-2040

Email: jdelgado@iupui.edu

This is the author's manuscript of the article published in final edited form as:

Delgado-Calle, J. (2017). Osteocytes and Their Messengers as Targets for the Treatment of Multiple Myeloma. Clinical Reviews in Bone and Mineral Metabolism, 15(1), 49-56. https://doi.org/10.1007/s12018-017-9227-7 


\begin{abstract}
Osteocytes, the most abundant cells in bone, orchestrate the function of osteoblasts and osteocytes to control physiological bone homeostasis. Accumulating evidence demonstrates that alteration of osteocyte function underlies the pathophysiology of several skeletal disorders, and therapeutic targeting of factors produced by these cells improves skeletal health. Despite the advances in the knowledge of osteocyte biology, the contribution of these cells to the damaging effects of cancer in bone is practically unknown. Multiple myeloma is a plasma cell malignancy characterized by the presence of skeletal lesions and severe bone pain. Recent findings suggest that myeloma cells educate osteocytes to generate a microenvironment that is conducive to tumor progression, skeletal destruction, and bone pain. This review features some of these investigations and discusses the potential of targeting osteocytic pathways and osteocyte messengers for the treatment of multiple myeloma.
\end{abstract}


Keywords: Osteocytes, myeloma, Sclerostin, Notch signaling, Rankl, apoptosis 


\section{Introduction}

Multiple myeloma (MM) is a B-cell neoplasm characterized by the growth of monoclonal plasma cells in the bone marrow. This debilitating malignancy usually presents with overproduction of monoclonal immunoglobulins, severe bone pain, pathologic fractures, weakness, anemia, infection, hypercalcemia, spinal cord compression, or renal failure [1-3]. MM is the second most common hematologic malignancy in the United States, representing $1.4 \%$ of all new cancers, with around 30,000 new cases and 12,500 people dying of this disease in 2016, as estimated by The American Cancer Society (https://cancerstatisticscenter.cancer.org). MM is most frequently diagnosed among people aged 65-74, more common in men than women, and is twice as frequent among African American descendants as it is in other racial groups [4].

MM has the highest incidence of bone involvement among malignant diseases. Skeletal manifestations are the most prominent source of pain and have devastating clinical effects, increasing the morbidity of MM patients. It is estimated that about $70 \%$ of $\mathrm{MM}$ patients have evidence of skeletal lesions at diagnosis, and this percentage increases to $90 \%$ in patients with advanced disease [2]. Further, up to 60\% of MM patients develop a pathological fracture during the course of the disease. Importantly, MM patients with pathologic fractures have a $20 \%$ increased risk of death [5]. MM bone disease (MMBD) is characterized by the development of focal skeletal lesions in areas close to active MM cells, while normal bone remodeling exists in bone areas without tumor involvement [6]. These localized osteolytic lesions result from exacerbated bone resorption and protracted suppression of new bone formation, due to the uncoupled and imbalanced activity of osteoblasts, bone-forming cells, and osteoclasts, bone-resorbing cells [7;8]. As a consequence, although these bone lesions stop progressing with anti-myeloma therapy, they rarely heal, even in patients in complete remission. 
MM is highly dependent on the bone/bone marrow microenvironment, and interactions between tumor cells and other cells present in this compartment are considered an important pathogenic factor in MM [9-11]. Under physiological conditions, bone remodeling takes place in a balanced manner within structures known as 'bone remodeling compartments' (BRCs) [12-14]. The BRC is often disrupted in MM patients, thus allowing MM cells to establish close ties with cells present in the MM microenvironment [15]. These interactions are key for the growth and survival of MM cells and the progression of MMBD [1;8;10]. The contribution of tumor cells, osteoclasts, osteoblasts, stromal cells, and immune cells to tumor growth and MMBD is well known. However, there is little information about whether osteocytes, 95\% of the cells present in bone and key regulators of bone homeostasis [16], contribute to the progression of myeloma and the associated bone disease. This review summarizes the current knowledge regarding the role of osteocytes in MM and examines the potential of targeting osteocytes and their derived factors as a novel therapeutic approach to treat MM.

\section{Osteocytes: key regulators of bone homeostasis.}

Osteocytes, the longest-lived of all bone cells, are fully differentiated osteoblasts that become entombed by matrix during the process of bone formation [17]. Although originally described as inactive quiescent cells, now we know that osteocytes actively control bone homeostasis [18;19]. Osteocytes have multiple cytoplasmic projections that run through canaliculi in the bone and connect them with other surrounding osteocytes and cells in the bone surface [17]. This canalicular system allows osteocytes to sense and transduce mechanical forces, as well as coordinate the function of osteoblasts and osteoclasts by mechanisms including direct cell-to-cell interaction and secretion of paracrine factors [16-20]. 
Osteocytes have a critical role in the regulation of bone formation by sending inhibitory signals that decrease the bone forming activity of osteoblasts. Osteocytes are major producers of antagonists of the Wnt/ $\beta$-catenin signaling, a pathway that when activated increases bone mass mainly due to increased osteoblast survival and bone formation, but also inhibits osteoclastogenesis by upregulating the expression of osteoprotegerin (OPG) in osteoblasts and osteocytes [21]. Wnt proteins bind to Frizzled receptors and to the co-receptors low-density lipoprotein receptor-related protein (LRP) 5 or 6 , to stabilize $\beta$-catenin protein and activate the transcription of target genes involved in osteoblasts differentiation, survival, and matrix synthesis. Osteocytes secrete Dickkopf-related protein 1 (DKK-1) and Sclerostin, the product of the gene SOST, to compete with Wnt ligands to bind to LRP 5 and 6, thus preventing Wnt signaling activation and slowing bone formation by osteoblasts [22-24]. Consistent with the osteo-anabaolic effects of canonical Wnt signaling, genetic and pharmacologic inhibition of DKK-1 or Sost/Sclerostin, in both humans and preclinical models, increases bone mass largely due to increased bone formation, with a component of decreased bone resorption [25-30]. However, activation of this pathway in osteoblasts induces bone gain due to a reduction in bone resorption [31]. In contrast, our group has recently demonstrated that the bone gain induced by activation of Wnt/ $\beta$-catenin signaling in osteocytes is due to increased bone formation [32]. These results suggest that, in addition to the paracrine effects of these inhibitors in osteoblasts and osteoclasts, they might also have autocrine effects in osteocytes.

Osteocytes also control bone resorption by producing and secreting pro- and antiosteoclastogenic cytokines. In the adult skeleton, osteocytes are the main source of the Receptor activator of nuclear factor kappa-B ligand (RANKL), a cytokine essential for osteoclast differentiation and survival [33-35]. Osteocytes also express OPG, a soluble decoy that blocks the 
binding of RANKL to its receptor, receptor activator of nuclear factor kappa-B (RANK) [36]. OPG is a Wnt target gene, thus osteocyte-mediated modulation of Wnt/ $\beta$-catenin signaling can also impact bone resorption [31;37]. Further, emerging evidence supports that Sclerostin, in a Wnt signaling independent fashion, can also modulate the expression of RANKL in osteocytes [22;32;38].

Osteocyte death is often accompanied by increased osteoclastogenesis in nearby bone, and osteocyte apoptosis is temporally and spatially associated with local osteoclastic resorption in several mouse models [19;39;40]. This evidence supports that apoptotic osteocytes trigger a cascade of events that directs osteoclasts to particular areas of the skeleton, starting a process known as "targeted" bone remodeling. Although the mechanism(s) underlying targeted remodeling are incompletely understood, it has been shown that apoptotic osteocytes express higher levels of RANKL (or stimulate the expression of this gene in surrounding osteocytes), release ATP, as well as other factors that could mediate the recruitment and differentiation of osteoclast precursors [41-45].

Our understanding of the function of osteocytes as regulators of bone homeostasis has increased greatly in the last two decades. Now we have extensive evidence demonstrating that genetic mutations in osteocytic genes have dramatic effects in the human skeleton [21;46], and that dysregulation of osteocyte function and alteration of osteocyte lifespan underlies the pathophysiology of several skeletal disorders [18]. As a result, several therapeutic approaches that target osteocytes and their messengers have been recently developed and shown beneficial skeletal outcomes in osteoporotic patients [17;18;22], demonstrating that osteocytes are a valuable target to combat bone diseases. 


\section{Osteocytes and the multiple myeloma microenvironment.}

The contribution of osteocytes to cancer in bone, and in particular to MM, is just starting to emerge. Over the last years accumulating evidence supports that myeloma cells use osteocytes to their advantage to generate a microenvironment that is conducive to tumor growth, skeletal destruction, and bone pain (Figure 1).

First evidence suggesting a potential role of osteocytes in myeloma was provided by Giuliani and colleagues, who found more apoptotic osteocytes in MM patients compared to healthy subjects or patients with monoclonal gammopathy of undetermined significance (MGUS) [47]. Consistent with this finding, we recently reported in a murine model of myeloma that the number of apoptotic osteocytes is increased in bone areas infiltrated with myeloma cells [48]. Mechanistic studies demonstrated that cell-to-cell interaction between osteocytes and myeloma cells, as well as myeloma-derived factors, are responsible for the decrease in osteocyte viability in the MM microenvironment [48]. Physical interactions between myeloma cells and osteocytes activate Notch signaling in osteocytes, a pathway that regulates proliferation and programmed cell death in several cell types [49], triggering caspase-3 mediated apoptosis [48]. In addition, accumulation of tumor necrosis factor $\alpha(\mathrm{TNF} \alpha)$ secreted by myeloma cells sustains/amplifies osteocyte apoptosis, a second mechanism by which these cancer cells shorten the lifespan of osteocytes [48]. Recent findings suggest that myeloma cells can also stimulate osteocyte apoptosis by inducing autophagy [50], a tightly regulated process of self-degradation that protects from oxidative stress stimuli or aging [51].

Consistent with the role of apoptotic osteocytes in targeting bone resorption to particular areas of the bone in other skeletal diseases [39;40], Giuliani and colleagues found a positive correlation between death osteocytes and the number of osteoclasts in bone samples from myeloma 
patients [47]. Further, in vitro experiments performed in our laboratory showed that apoptosis enhances the ability of osteocytes to attract osteoclast precursors, and increases the expression of RANKL to stimulate osteoclastogenesis [47;48]. It is also possible that other factors released by osteocytes, such as interleukin (IL)-11, play a role in the increased resorption that accompanies the growth of myeloma cells in the bone/bone marrow [47]. Taken together, these results support that osteocytes, and in particular apoptotic osteocytes, contribute to the exaggerated bone resorption in MM.

Not only osteocyte viability, but also osteocytic gene expression is altered in MM. Osteocytes overproduce Sost/Sclerostin in MM-colonized bones, leading to Wnt signaling inhibition and subsequently impairing osteoblast differentiation [48]. These data support that osteocytes also participate in the suppression of osteoblast differentiation and new bone formation by increasing the levels of Sclerostin in the microenvironment. In addition, the expression of the Wht target gene OPG is also decreased in osteocytes exposed to myeloma cells, thus increasing even further the RANKL/OPG and their osteoclastogenic potential [48]. Moreover, the Notch receptor repertoire of osteocytes is altered in bones with MM involvement, with Notch receptor 3 (NOTCH R3) rapidly increasing upon interaction with myeloma cells [48].

Osteocytes also seem to fuel the growth of myeloma cells by direct and indirect mechanisms. We recently showed that direct cell-to-cell contact with osteocytes reciprocally activates Notch signaling in myeloma cells to stimulate tumor growth [48]. Autocrine and paracrine [interactions with stromal cells] activation of Notch signaling through the Notch receptor 1 and 2 stimulates the proliferation of myeloma cells [52-55]. However, we found that the expression of NOTCH R3 and 4 in myeloma cells is markedly increased and induced, respectively, upon physical contact with osteocytes [48]. These results suggest that bidirectional communication 
between osteocytes and myeloma cells stimulates the proliferation of myeloma cells via NOTCH R3 and/or R4 signaling. Further, because apoptotic osteocytes increase bone resorption, it is possible that these cells enhanced tumor growth driven by factors released due to osteoclastic resorption. Moreover, fibroblast growth factor 23 (FGF23) produced by osteocytes induces myeloma proliferation by binding to FGF23 receptors and the co-receptor Klotho in myeloma cells [56]. Recent findings suggest that adipocytes support the proliferation and dissemination of MM [57;58]. Activation of Wnt signaling favors mesenchymal stromal cell differentiation to osteoblasts, and blocks adipogenic differentiation [21], while Sclerostin-mediated inhibition of this pathway stimulates adipocyte differentiation in vitro and in vivo [59-61]. Thus, it is possible that osteocyte-secreted Sclerostin stimulates the differentiation of mesenchymal precursors towards the adipogeneic lineage, and therefore support myeloma growth [62].

Bone pain is one of the most common complications in MM and a major cause of morbidity and diminished quality of life. The mechanisms responsible for MM-induced bone pain are poorly understood, but may include interactions between myeloma cells, bone cells, and peripheral nerves [63]. Recent data suggest that osteocytes can communicate with sensory nerves and potentially play an important role in MM-induced bone pain [64]. In vivo, osteocyte physically interact with sensory nerves, and in vitro studies show that osteocytes induce neurite sprouting and sensory nerve excitation [64]. Communication between osteocytes and sensory nerves appears to be mediated by gap junctions. Treatment with GAP27, a selective gap junction blocker, suppresses MM-induced bone pain in vivo. More importantly, bone pain induced by myeloma is reduced in mice lacking connexin 43 (Cx43) in osteocytes compared to control mice [64]. Taken together, these results demonstrate that osteocyte and sensory nerves are in intimate physical contact in 
bone, and that they exchange small molecules via Cx43 to induce sensory nerve excitation and bone pain.

\section{Osteocytes as targets for the treatment of multiple myeloma.}

Recent advances in the treatment of MM have increased patient survival [1]. Yet, current available anti-myeloma regimens have minor effects on bone repair [65]. Bisphosphonates, a class of drugs that prevent bone loss but suppress bone remodeling, are the mainstay of therapy to treat MMBD [66]. In addition, glucocorticoids [GC] are a frequent component of MM treatment in combination with proteasome inhibitors and/or immunomodulatory drugs [67-69]. However, GC cause bone loss, by increasing bone resorption and reducing formation, and also induce muscle atrophy [70;71]. GC, similar to myeloma cells, exert their bone effects, in part, by inducing osteocyte apoptosis, which in turn stimulates resorption, and increasing the expression of Sost/Sclerostin to inhibit bone formation [71;72]. Therefore, MM patients live longer but continue to suffer from increasingly devastating skeletal sequelae. Thus, new therapeutic regimens that simultaneously decrease tumor progression and improve MMBD are sorely needed.

New evidence suggest that targeting osteocyte-myeloma cell interactions represents a promising treatment strategy for the treatment of MMBD, as well as potentially suppress myeloma growth. The Notch signaling pathway has gained growing interest in the recent years because of the dysregulation of several Notch components in myeloma cells and its role in several aspects of the disease [73;74]. The most widely drugs used to block Notch signaling are inhibitors of the gamma-secretase (GSIs), a complex responsible for the cleavage of the intracellular domain of the Notch receptors [75;76]. Our in vitro data suggests that GSIs could protect osteocytes from 
apoptosis induced by myeloma cells, thus preventing osteoclast recruitment, as well as decrease osteocyte stimulation of myeloma proliferation [48]. However, GSIs have significant toxicity and cause several adverse events, among others gut toxicity and skin disorders [73;74]. Thus, targeting specific components of the Notch pathway has become an attractive approach to avoid the offtarget effects of GSIs. In this regard, neutralizing antibodies have been generated to block the Notch ligands Delta-1 and Jagged-1 [73;74], which are currently being studied in preclinical models. The studies on osteocytes mentioned above suggest that pharmacological inhibition of specific Notch receptors, in particular NOTCH R3 and 4, may also represent a promising treatment strategy in MM. Future studies to investigate this possibility and to identify relevant Notch ligands/receptors on osteocytes and myeloma cells are warranted.

Sclerostin and DKK-1 levels are elevated in the sera of MM patients and correlate with reduced osteoblast function and poor patient survival [77-79], suggesting that these Wnt antagonists contribute to and may be a therapeutic target for the treatment of MMBD. DKK1 is expressed by myeloma cells, as well as by osteocytes, and DKK-1-neutralizing antibodies abolish suppression of osteoblastogenesis and have variable effects on myeloma growth [79;80]. Similarly, recent data from our laboratory show that genetic deletion of Sost prevents MM-induced bone loss in a mouse model that recapitulates the early course of MM [81]. More importantly, we found that treatment with Scl-Ab reduced osteolysis and increased cancellous bone volume in immunocompetent mice in the late stages of MM [81]. Further, we showed that inhibition of Sost/Sclerostin did not alter MM cell growth in vivo or in vitro, and preserved the efficacy of antimyeloma drugs in vitro [81]. Consistent with these findings, Scl-Ab administration shortly after injection of myeloma cells also prevented the decrease in cancellous bone mass in the early course of MM, with no effects on tumor burden [78;82]. These results demonstrate that overproduction 
of Sclerostin by osteocytes contributes to MMBD and suggest that combination of Scl-Ab with anti-tumor drugs represents a potential therapeutic approach to simultaneously decrease myeloma progression and improve MMBD.

Actions on osteocytes could also mediate some of the beneficial effects observed with drugs used to treat MM. Soluble RANKL levels are elevated in the sera of MM patients and positively correlate with disease activity [83]. RANKL neutralization delays myeloma progression in mice [84], and reduces bone resorption in myeloma patients [85]. Several cells present in the MM microenvironment express RANKL, including osteocytes, which are considered the major source of this molecule in adult bone [34]. Although the specific contribution of osteocyte-derived RANKL to the increased osteolysis in MM remains to be determined, it is possible that some of the beneficial effects observed with monoclonal antibodies against RANKL derived from neutralization of osteocytic RANKL. The use of proteasome inhibitors has emerged as an important therapeutic strategy for the treatment of MM [3]. Bortezomib is currently approved for the treatment of myeloma and improves MMBD through actions on osteoblasts [86-88]. Bortezomib induces mitochondrial injury and apoptosis in myeloma cells, but also inhibits MMinduced osteocyte autophagy, suggesting that osteocytes are also direct targets of this drug [50]. Therefore some of the clinical benefits observed with Bortezomib therapy could arise from a preservation of osteocyte viability.

\section{Conclusions and future directions.}

Advances over the last years provided clear evidence that osteocytes are part of the MM microenvironment and contribute to the deleterious effects of myeloma cells in the skeleton. Now 
we know that myeloma cells instruct osteocytes to support tumor proliferation, increase bone resorption and suppress bone formation, and that in bones bearing myeloma osteocytes stimulate sensory nerves to promote bone pain. Importantly, new evidence suggests that osteocytes could also participate in the growth of other cancers in bone [89;90]. The discovery of some of the pathways and molecular messengers altered in osteocytes present in the cancer niche has the potential to guide the development of new therapeutic regimens for the treatment of myeloma and the associated bone disease, and eventually other cancers. Future investigations are warranted to reveal the specific contribution of osteocytes to the progression of cancer in bone, and to identify new osteocytic molecules and mechanisms that can be exploited for the management of cancer in bone. 


\section{Figure captions}

Figure 1. Osteocytes contribute to generate a microenvironment that is conducive to tumor growth, skeletal destruction, and bone pain. In MM-colonized bone, physical communication between osteocytes and myeloma cells activates bidirectional Notch signaling, resulting in osteocyte apoptosis and increased myeloma proliferation. Apoptotic osteocytes express RANKL to target osteoclasts to the area and initiate bone resorption. In addition, myeloma cells produced DKK-1 and reprogram osteocytes to overproduce Sclerostin, which in turn decreases Wnt signaling, resulting in the downregulation of OPG expression and inhibition of osteoblast differentiation. Further, osteocytes, via Cx43, exchange factors with sensory nerves to induce sensory nerve excitation and bone pain. 


\section{Acknowledgements}

Research reported in this manuscript was supported by the National Institutes of Health Grants (Indiana-CTSI P30, 1R21CA179017-02, R01AR059679, R01AR059357, R01 DK076007, and S10-RR023710), the Veteran's Administration, the International Bone and Mineral Society Gideon and Sevgi Rodan Fellowship, the American Society of Hematology Scholar Award, and the International Myeloma Foundation Brian D. Novis Junior Research Grant. The author thanks Drs. Teresita Bellido and G. David Roodman for their insightful comments and suggestions.

\section{Compliance with Ethical Standards}

- Conflict of interest: Jesus Delgado-Calle declares that has no conflict of interest.

- Animal and Human Studies: This article includes studies with mice performed by the author. All procedures performed in these studies were approved by the Institutional Animal Care and Use Committee of the Indiana University School of Medicine. This article does not include any studies with humans performed by the author. 
- Informed consent: Not applicable.

\section{Reference List}

[1] Bianchi G, Munshi NC. Pathogenesis beyond the cancer clone[s] in multiple myeloma. Blood 2015; 125[20]:3049-3058.

[2] Greenberg AJ, Rajkumar SV, Therneau TM, Singh PP, Dispenzieri A, Kumar SK. Relationship between initial clinical presentation and the molecular cytogenetic classification of myeloma. Leukemia 2014; 28[2]:398-403.

[3] Rajkumar SV. Myeloma today: Disease definitions and treatment advances. Am J Hematol 2016; 91[1]:90-100.

[4] Benjamin M, Reddy S, Brawley OW. Myeloma and race: a review of the literature. Cancer Metastasis Rev 2003; 22[1]:87-93.

[5] Saad F, Lipton A, Cook R, Chen YM, Smith M, Coleman R. Pathologic fractures correlate with reduced survival in patients with malignant bone disease. Cancer 2007; 110[8]:18601867.

[6] Valentin-Opran A, Charhon SA, Meunier PJ, Edouard CM, Arlot ME. Quantitative histology of myeloma-induced bone changes. Br J Haematol 1982; 52[4]:601-610.

[7] Terpos E, Berenson J, Raje N, Roodman GD. Management of bone disease in multiple myeloma. Expert Rev Hematol 2014; 7[1]:113-125.

[8] Silbermann R, Roodman GD. Myeloma bone disease: Pathophysiology and management. J Bone Oncol 2013; 2[2]:59-69.

[9] Roodman GD. Pathogenesis of myeloma bone disease. J Cell Biochem 2010; 109[2]:283291.

[10] Roodman GD. Targeting the bone microenvironment in multiple myeloma. J Bone Miner Metab 2010; 28[3]:244-250. 
[11] Roodman GD. Role of the bone marrow microenvironment in multiple myeloma. J Bone Miner Res 2002; 17[11]:1921-1925.

[12] Hauge EM, Qvesel D, Eriksen EF, Mosekilde L, Melsen F. Cancellous bone remodeling occurs in specialized compartments lined by cells expressing osteoblastic markers. J Bone Miner Res 2001; 16[9]:1575-1582.

[13] Eriksen EF, Eghbali-Fatourechi GZ, Khosla S. Remodeling and vascular spaces in bone. J Bone Miner Res 2007; 22[1]:1-6.

[14] Andersen TL, Sondergaard TE, Skorzynska KE, gnaes-Hansen F, Plesner TL, Hauge EM et al. A physical mechanism for coupling bone resorption and formation in adult human bone. Am J Pathol 2009; 174[1]:239-247.

[15] Andersen TL, Soe K, Sondergaard TE, Plesner T, Delaisse JM. Myeloma cell-induced disruption of bone remodelling compartments leads to osteolytic lesions and generation of osteoclast-myeloma hybrid cells. Br J Haematol 2010; 148[4]:551-561.

[16] Bonewald LF. The Amazing Osteocyte. J Bone Miner Res 2011; 26[2]:229-238.

[17] Plotkin LI, Bellido T. Osteocytic signalling pathways as therapeutic targets for bone fragility. Nat Rev Endocrinol 2016; 12[10]:593-605.

[18] Delgado-Calle J, Bellido T. Osteocytes and Skeletal Pathophysiology. Curr Mol Biol Rep 2015; 1[4]:157-167.

[19] Bellido T. Osteocyte-Driven Bone Remodeling. Calcif Tissue Int 2013; 94[1]:25-34.

[20] Dallas SL, Prideaux M, Bonewald LF. The osteocyte: an endocrine cell ... and more. Endocr Rev 2013; 34[5]:658-690.

[21] Baron R, Kneissel M. WNT signaling in bone homeostasis and disease: from human mutations to treatments. Nat Med 2013; 19[2]:179-192.

[22] Delgado-Calle J, Sato AY, Bellido T. Role and mechanism of action of sclerostin in bone. Bone 2016; pii: S8756-3282[16]30297-30306.

[23] Poole KE, Van Bezooijen RL, Loveridge N, Hamersma H, Papapoulos SE, Lowik CW et al. Sclerostin is a delayed secreted product of osteocytes that inhibits bone formation. FASEB J 2005; 19[13]:1842-1844.

[24] Van Bezooijen RL, Roelen BA, Visser A, Wee-Pals L, de Wilt E, Karperien M et al. Sclerostin is an osteocyte-expressed negative regulator of bone formation, but not a classical BMP antagonist. J Exp Med 2004; 199[6]:805-814.

[25] Ominsky MS, Boyce RW, Li X, Ke HZ. Effects of sclerostin antibodies in animal models of osteoporosis. Bone 2016; pii: S8756-3282[16]30315-30325. 
[26] Ke HZ, Richards WG, Li X, Ominsky MS. Sclerostin and Dickkopf-1 as Therapeutic Targets in Bone Diseases. Endocr Rev 2012; 33[5]:747-783.

[27] Li X, Ominsky MS, Niu QT, Sun N, Daugherty B, D'Agostin D et al. Targeted deletion of the sclerostin gene in mice results in increased bone formation and bone strength. J Bone Miner Res 2008; 23[6]:860-869.

[28] Glantschnig H, Hampton RA, Lu P, Zhao JZ, Vitelli S, Huang L et al. Generation and selection of novel fully human monoclonal antibodies that neutralize Dickkopf-1 [DKK1] inhibitory function in vitro and increase bone mass in vivo. J Biol Chem 2010; 285[51]:40135-40147.

[29] Li J, Sarosi I, Cattley RC, Pretorius J, Asuncion F, Grisanti M et al. Dkk1-mediated inhibition of Wnt signaling in bone results in osteopenia. Bone 2006; 39[4]:754-766.

[30] McClung MR, Grauer A, Boonen S, Bolognese MA, Brown JP, ez-Perez A et al. Romosozumab in Postmenopausal Women with Low Bone Mineral Density. N Engl J Med 2014; 370[5]:412-420.

[31] Glass DA, Bialek P, Ahn JD, Starbuck M, Patel MS, Clevers H et al. Canonical Wnt signaling in differentiated osteoblasts controls osteoclast differentiation. Dev Cell 2005; 8[5]:751-764.

[32] Tu X, Delgado-Calle J, Condon KW, Maycas M, Zhang H, Carlesso N et al. Osteocytes mediate the anabolic actions of canonical Wnt/b-catenin signaling in bone. Proc Natl Acad Sci U S A 2015; 112[5]:E478-E486.

[33] Xiong J, Piemontese M, Onal M, Campbell J, Goellner JJ, Dusevich V et al. Osteocytes, not Osteoblasts or Lining Cells, are the Main Source of the RANKL Required for Osteoclast Formation in Remodeling Bone. PLoS ONE 2015; 10[9]:e0138189.

[34] O'Brien CA, Nakashima T, Takayanagi H. Osteocyte control of osteoclastogenesis. Bone 2013; 54[2]:258-263.

[35] Xiong J, Onal M, Jilka RL, Weinstein RS, Manolagas SC, O'Brien CA. Matrix-embedded cells control osteoclast formation. Nat Med 2011; 17[10]:1235-1241.

[36] Boyce BF, Xing L. Biology of RANK, RANKL, and osteoprotegerin. Arthritis Res Ther 2007; 9 Suppl 1:S1.

[37] Kramer I, Halleux C, Keller H, Pegurri M, Gooi JH, Weber PB et al. Osteocyte Wnt/betacatenin signaling is required for normal bone homeostasis. Mol Cell Biol 2010; 30[12]:3071-3085.

[38] Wijenayaka AR, Kogawa M, Lim HP, Bonewald LF, Findlay DM, Atkins GJ. Sclerostin stimulates osteocyte support of osteoclast activity by a RANKL-dependent pathway. PLoS ONE 2011; 6[10]:e25900. 
[39] Plotkin LI. Apoptotic osteocytes and the control of targeted bone resorption. Curr Osteoporos Rep 2014; 12[1]:121-126.

[40] Schaffler MB, Cheung WY, Majeska R, Kennedy O. Osteocytes: Master Orchestrators of Bone. Calcif Tissue Int 2013; 94[1]:5-24.

[41] Cabahug-Zuckerman P, Frikha-Benayed D, Majeska RJ, Tuthill A, Yakar S, Judex S et al. Osteocyte Apoptosis Caused by Hindlimb Unloading is Required to Trigger Osteocyte RANKL Production and Subsequent Resorption of Cortical and Trabecular Bone in Mice Femurs. J Bone Miner Res 2016; 31[7]:1356-1365.

[42] Plotkin LI, de Gortazar AR, Davis HM, Condon KW, Gabilondo H, Maycas M et al. Inhibition of Osteocyte Apoptosis Prevents the Increase in Osteocytic RANKL but it does not Stop Bone Resorption or the Loss of Bone Induced by Unloading. J Biol Chem 2015; 290[31]:18934-18941.

[43] Sandilos JK, Chiu YH, Chekeni FB, Armstrong AJ, Walk SF, Ravichandran KS et al. Pannexin 1, an ATP release channel, is activated by caspase cleavage of its pore-associated C-terminal autoinhibitory region. J Biol Chem 2012; 287[14]:11303-11311.

[44] Chekeni FB, Elliott MR, Sandilos JK, Walk SF, Kinchen JM, Lazarowski ER et al. Pannexin 1 channels mediate 'find-me' signal release and membrane permeability during apoptosis. Nature 2010; 467[7317]:863-867.

[45] Cheung WY, Fritton JC, Morgan SA, Seref-Ferlengez Z, Basta-Pljakic J, Thi MM et al. Pannexin-1 and P2X7-Receptor Are Required for Apoptotic Osteocytes in Fatigued Bone to Trigger RANKL Production in Neighboring Bystander Osteocytes. J Bone Miner Res 2016; 31[4]:890-899.

[46] Loots GG, Kneissel M, Keller H, Baptist M, Chang J, Collette NM et al. Genomic deletion of a long-range bone enhancer misregulates sclerostin in Van Buchem disease. Genome Res 2005; 15[7]:928-935.

[47] Giuliani N, Ferretti M, Bolzoni M, Storti P, Lazzaretti M, Dalla PB et al. Increased osteocyte death in multiple myeloma patients: role in myeloma-induced osteoclast formation. Leukemia 2012; 26[6]:1391-1401.

[48] Delgado-Calle J, Anderson J, Cregor MD, Hiasa M, Chirgwin JM, Carlesso N et al. Bidirectional Notch signaling and osteocyte-derived factors in the bone marrow microenvironment promote tumor cell proliferation and bone destruction in multiple myeloma. Cancer Res 2016; 76[5]:1089-1100.

[49] Bray SJ. Notch signalling: a simple pathway becomes complex. Nat Rev Mol Cell Biol 2006; 7[9]:678-689.

[50] Toscani D, Palumbo C, Dalla PB, Ferretti M, Bolzoni M, Marchica V et al. The Proteasome Inhibitor Bortezomib Maintains Osteocyte Viability in Multiple Myeloma Patients by 
Reducing Both Apoptosis and Autophagy: A New Function for Proteasome Inhibitors. J Bone Miner Res 2016; 31[4]:815-827.

[51] Cuervo AM. Breaking down autophagy. Trends Cell Biol 2016; 6[9]:637-638.

[52] Nefedova Y, Cheng P, Alsina M, Dalton WS, Gabrilovich DI. Involvement of Notch-1 signaling in bone marrow stroma-mediated de novo drug resistance of myeloma and other malignant lymphoid cell lines. Blood 2004; 103[9]:3503-3510.

[53] Xu D, Hu J, Xu S, De BE, Menu E, Van CB et al. Dll1/Notch activation accelerates multiple myeloma disease development by promoting CD138+ MM-cell proliferation. Leukemia 2012; 26[6]:1402-1405.

[54] Jundt F, Probsting KS, Anagnostopoulos I, Muehlinghaus G, Chatterjee M, Mathas S et al. Jagged1-induced Notch signaling drives proliferation of multiple myeloma cells. Blood 2004; 103[9]:3511-3515.

[55] Chiron D, Maiga S, Descamps G, Moreau P, Le GS, Marionneau S et al. Critical role of the NOTCH ligand JAG2 in self-renewal of myeloma cells. Blood Cells Mol Dis 2012; 48[4]:247-253.

[56] Suvannasankha A, Tompkins DR, Edwards DF, Petyaykina KV, Crean CD, Fournier PG et al. FGF23 is elevated in multiple myeloma and increases heparanase expression by tumor cells. Oncotarget 2015; 6[23]:19647-19660.

[57] Trotter TN, Gibson JT, Sherpa TL, Gowda PS, Peker D, Yang Y. Adipocyte-Lineage Cells Support Growth and Dissemination of Multiple Myeloma in Bone. Am J Pathol 2016; 186[11]:3054-3063.

[58] Yu W, Cao DD, Li QB, Mei HL, Hu Y, Guo T. Adipocytes secreted leptin is a pro-tumor factor for survival of multiple myeloma under chemotherapy. Oncotarget 2016; doi: 10.18632/oncotarget.13342.

[59] Ukita M, Yamaguchi T, Ohata N, Tamura M. Sclerostin Enhances Adipocyte Differentiation in 3T3-L1 Cells. J Cell Biochem 2016; 117[6]:1419-1428.

[60] Fulzele K, Lai F, Dedic C, Saini V, Uda Y, Shi C et al. Osteocyte-Secreted Wnt Signaling Inhibitor Sclerostin Contributes to Beige Adipogenesis in Peripheral Fat Depots. J Bone Miner Res 2016; doi: 10.1002/jbmr.3001.

[61] Chandra A, Lin T, Young T, Tong W, Ma X, Tseng WJ et al. Suppression of Sclerostin Alleviates Radiation-Induced Bone Loss by Protecting Bone-Forming Cells and Their Progenitors Through Distinct Mechanisms. J Bone Miner Res 2016; doi: 10.1002/jbmr.2996.

[62] McDonald MM, Fairfield H, Falank C, Reagan MR. Adipose, Bone, and Myeloma: Contributions from the Microenvironment. Calcif Tissue Int 2016; doi: 10.1007/s00223016-0162-2 
[63] Yoneda T, Hiasa M, Nagata Y, Okui T, White FA. Acidic microenvironment and bone pain in cancer-colonized bone. Bonekey Rep 2015; 4:690.

[64] Hiasa M, Okui T, Delgado-Calle J, Bellido T, Roodman GD, White F et al. Osteocytes Mediate Bone Pain Through Cell-Cell Communication with Sensory Neurons via Connexin 43. Journal of Bone and Mineral Research 2016; 31[Supl. 1]:S40.

[65] Silbermann R, Roodman GD. Bone effects of cancer therapies: pros and cons. Curr Opin Support Palliat Care 2011; 5[3]:251-257.

[66] Papamerkouriou YM, Kenanidis E, Gamie Z, Papavasiliou K, Kostakos T, Potoupnis M et al. Treatment of multiple myeloma bone disease: experimental and clinical data. Expert Opin Biol Ther 2015; 15[2]:213-230.

[67] Chauhan D, Hideshima T, Pandey P, Treon S, Teoh G, Raje N et al. RAFTK/PYK2dependent and -independent apoptosis in multiple myeloma cells. Oncogene 1999; 18[48]:6733-6740.

[68] Chauhan D, Pandey P, Ogata A, Teoh G, Treon S, Urashima M et al. Dexamethasone induces apoptosis of multiple myeloma cells in a JNK/SAP kinase independent mechanism. Oncogene 1997; 15[7]:837-843.

[69] Eshaghian S, Berenson JR. Multiple myeloma: improved outcomes with new therapeutic approaches. Curr Opin Support Palliat Care 2012; 6[3]:330-336.

[70] Weinstein RS. Clinical practice. Glucocorticoid-induced bone disease. N Engl J Med 2011; 365[1]:62-70.

[71] Sato AY, Cregor M, Delgado-Calle J, Condon KW, Allen MR, Peacock M et al. Protection from Glucocorticoid-Induced Osteoporosis by Anti-Catabolic Signaling in the Absence of Sost/Sclerostin. J Bone Miner Res 2016; 1[10]:1791-1802.

[72] O'Brien CA, Jia D, Plotkin LI, Bellido T, Powers CC, Stewart SA et al. Glucocorticoids act directly on osteoblasts and osteocytes to induce their apoptosis and reduce bone formation and strength. Endocrinology 2004; 145[4]:1835-1841.

[73] Colombo M, Mirandola L, Platonova N, Apicella L, Basile A, Figueroa AJ et al. Notchdirected microenvironment reprogramming in myeloma: a single path to multiple outcomes. Leukemia 2013; 27[5]:1009-1018.

[74] Colombo M, Galletti S, Garavelli S, Platonova N, Paoli A, Basile A et al. Notch signaling deregulation in multiple myeloma: A rational molecular target. Oncotarget 2015; 6[29]:26826-26840.

[75] Zanotti S, Canalis E. Notch Signaling in Skeletal Health and Disease. Eur J Endocrinol 2013; 168[6]:R95-R103. 
[76] Hori K, Sen A, rtavanis-Tsakonas S. Notch signaling at a glance. J Cell Sci 2013; 126[Pt 10]:2135-2140.

[77] Terpos E, Christoulas D, Katodritou E, Bratengeier C, Gkotzamanidou M, Michalis E et al. Elevated circulating sclerostin correlates with advanced disease features and abnormal bone remodeling in symptomatic myeloma: reduction post-bortezomib monotherapy. Int J Cancer 2012; 131[6]:1466-1471.

[78] Eda H, Santo L, Wein MN, Hu DZ, Cirstea DD, Nemani N et al. Regulation of Sclerostin Expression in Multiple Myeloma by Dkk-1; A Potential Therapeutic Strategy for Myeloma Bone Disease. J Bone Miner Res 2016; 31[6]:1225-1234.

[79] Yaccoby S, Ling W, Zhan F, Walker R, Barlogie B, Shaughnessy JD, Jr. Antibody-based inhibition of DKK1 suppresses tumor-induced bone resorption and multiple myeloma growth in vivo. Blood 2007; 109[5]:2106-2111.

[80] Yaccoby S. Osteoblastogenesis and tumor growth in myeloma. Leuk Lymphoma 2010; 51[2]:213-220.

[81] Delgado-Calle J, Anderson J, Cregor MD, Zhou D, Plotkin LI, Bellido T, Roodman GD. Genetic Sost Deletion or Pharmacological Inhibition of Sclerostin Prevents Bone Loss and Decreases Osteolytic Lesions in Immunodeficient and Immunocompetent. J Bone Miner Res 2016; 31[Supl. 1]:S30.

[82] Reagan MR, McDonald M, Terry R, Pettitt J, Le L, Mohanty S et al. Anti-Sclerostin Treatment Prevents Multiple Myeloma Induced Bone Loss and Reduces Tumor Burden. Blood 2015; 126[23]:119.

[83] Jakob C, Goerke A, Terpos E, Sterz J, Heider U, Kuhnhardt D et al. Serum levels of totalRANKL in multiple myeloma. Clin Lymphoma Myeloma 2009; 9[6]:430-435.

[84] Sordillo EM, Pearse RN. RANK-Fc: a therapeutic antagonist for RANK-L in myeloma. Cancer 2003; 97[3 Suppl]:802-812.

[85] Vij R, Horvath N, Spencer A, Taylor K, Vadhan-Raj S, Vescio R et al. An open-label, phase 2 trial of denosumab in the treatment of relapsed or plateau-phase multiple myeloma. Am J Hematol 2009; 84[10]:650-656.

[86] Terpos E, Kleber M, Engelhardt M, Zweegman S, Gay F, Kastritis E et al. European Myeloma Network guidelines for the management of multiple myeloma-related complications. Haematologica 2015; 100[10]:1254-1266.

[87] Zangari M, Terpos E, Zhan F, Tricot G. Impact of bortezomib on bone health in myeloma: a review of current evidence. Cancer Treat Rev 2012; 38[8]:968-980.

[88] Giuliani N, Morandi F, Tagliaferri S, Lazzaretti M, Bonomini S, Crugnola M et al. The proteasome inhibitor bortezomib affects osteoblast differentiation in vitro and in vivo in multiple myeloma patients. Blood 2007; 110[1]:334-338. 
[89] Sottnik JL, Dai J, Zhang H, Campbell B, Keller ET. Tumor-induced pressure in the bone microenvironment causes osteocytes to promote the growth of prostate cancer bone metastases. Cancer Res 2015; 75[11]:2151-2158.

[90] Zhou JZ, Riquelme MA, Gu S, Kar R, Gao X, Sun L et al. Osteocytic connexin hemichannels suppress breast cancer growth and bone metastasis. Oncogene 2016; 35[43]:5597-5607. 


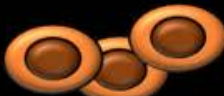

osteoblast

precursor

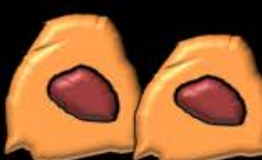

osteoblast

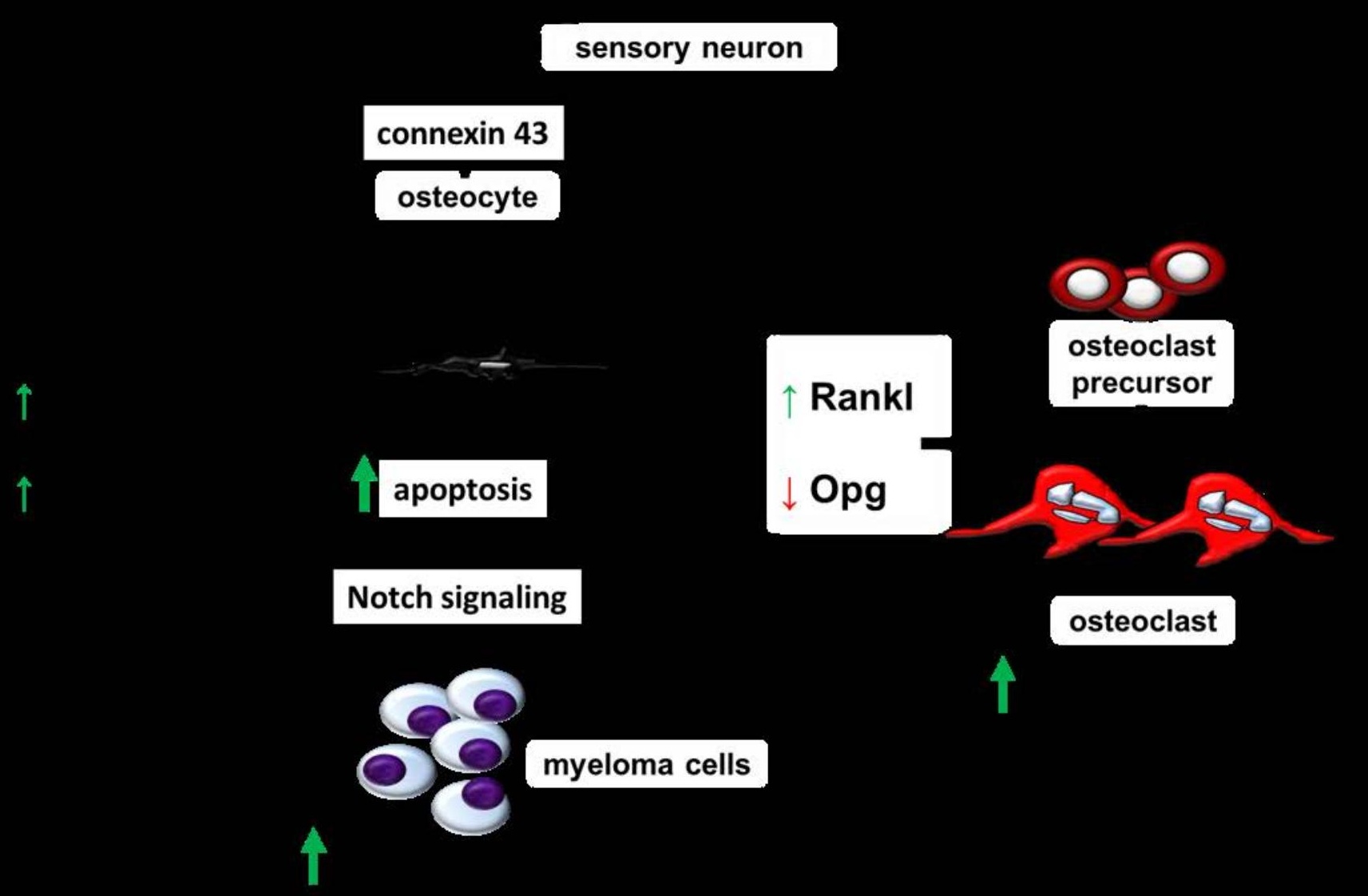

